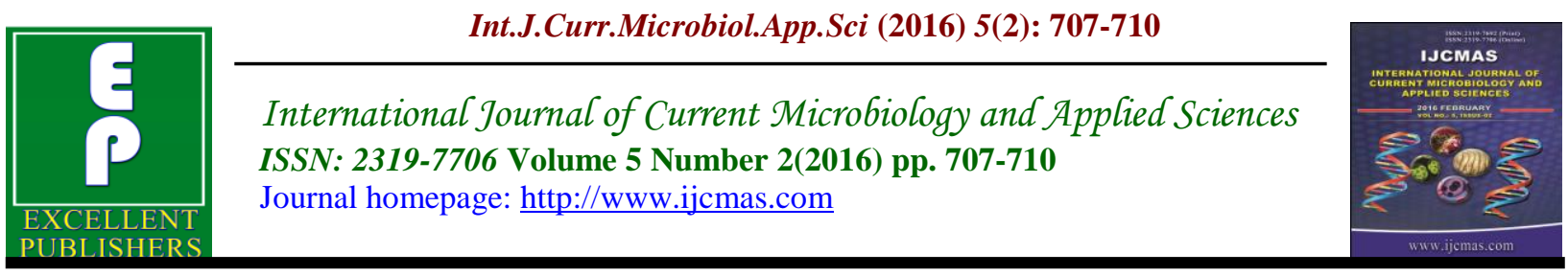

Original Research Article

doi: http://dx.doi.org/10.20546/ijcmas.2016.502.078

\title{
Study of Prevalence of Community Acquired Mycoplasma pneumoniae Infections at KIMS Hospital, Hubballi, India
}

\author{
N. Pramod Sambrani*, B. Asha Patil and A. Divya \\ Department of Microbiology, Karnataka Institute of Medical Sciences, \\ Hubballi, Karnataka, India \\ *Corresponding author
}

\begin{abstract}
A B S T R A C T
Keywords

Atypical pneumonia, Communityacquired pneumonia, Mycoplasma pneumoniae.

Article Info

Accepted:

28 January 2016

Available Online:

10, February 2016

Mycoplasma pneumoniae is a common and important pathogenic cause of community-acquired pneumonia presenting with fever and respiratory symptoms of varied severity. Therefore, laboratory diagnostic methods are particularly important for the diagnosis and treatment of atypical pneumonia. A study was conducted to determine the significance of this association and to estimate the prevalence of Community Acquired Mycoplasma Pneumonia by detecting IgM antibodies of Mycoplasma pneumoniae in the serum of patients attending the KIMS OPD, Hubballi with complaints of fever and respiratory symptoms. ${ }^{1}$ Serum samples of 82 patients were collected and tested for Mycoplasma pneumoniae IgM antibodies by using commercially obtained (Calbiotech) Mycoplasma pneumoniae IgM ELISA kit. Out of 82 serum samples tested for Mycoplasma pneumoniae-specific immunoglobulin $\mathrm{M}$ (IgM), 10 were positive accounting to $12.19 \%$. Detection of IgM antibodies by ELISA for precise and reliable diagnosis of Mycoplasma pneumoniae infections during the acute phases of disease indicating a possible use of ELISA as a valid diagnostic approach in early detection of Mycoplasma pneumoniae infections in patients with Community acquired pneumonia. ${ }^{2}$
\end{abstract}

\section{Introduction}

Mycoplasma pneumoniaeis a common respiratory pathogen that produce diseases of varied severity ranging from mild upper respiratory tract infection to severe atypical pneumonia. Although rarely fatal, $M$. pneumoniae is an important cause of acute respiratory tract infection, especially as a potential aetiology of the clinical entity termed "atypical pneumonia. ${ }^{3}$

The atypical respiratory bacterial pathogens Mycoplasma pneumoniae, Chlamydia pneumoniae and Legionella pneumophila are now recognised as a significant cause of acute respiratory-tract infections, but they remain colourless after Gram-staining and are difficult to identify by conventional bacterial culture tests. It is reported that patients with atypical pneumonia were more likely to have normal or reduced white bloodcell counts.However some published data showed that between atypical pneumonia and general bacterial pneumonia, there were no significant differences in the 
symptoms such as fever, cough, productive sputum and the sign of lung rales. Commonly prescribed antibiotics like betalactams are not effective for against Mycoplasama pneumonia. Therefore, laboratory detection methods in diagnosis and treatment of atypical pathogenic infection is particularly important ${ }^{4}$.

As most infections occur among outpatients hence the colloquial term walking pneumonia.

So the present study is undertaken to know the prevalance of infection caused Mycoplasma pneumoniae and age susceptibility in patients attending the OPD of KIMS, Hubballi.

\section{Subjects and Method}

\section{Study Design and Patients}

Under strict aseptic precautions, serum samples of 82 patients were collected between November 2015 and December 2015 in the KIMS OPD, Hubballi, Karnataka and a prospective study was conducted. All sera were tested for Mycoplasma pneumoniae IgM antibodies by using Mycoplasma pneumoniae IgM ELISA kit from Calbiotech. Test on all individual specimens were performed on the same day.

\section{Inclusion and Exclusion Criteria}

\section{Inclusion Criteria}

Patients attending KIMS OPD, Hubballi with complaints of fever for more than 5-7 days.

Patients attending KIMS OPD, Hubballi with respiratory complaints.

\section{Exclusion Criteria}

Patients diagnosed with other causes of fever.
Patients suffering from other causes of respiratory tract infection.

\section{Enzyme Immunoassay}

The Mycoplasma pneumoniae IgM antibodies were evaluated with Calbiotech IgM ELISA kit. Positive and Negative results were determined by the cut-off values. Each run was validated with the positive and negative controls included in the kits.

The results were expressed as an Antibody index $(\mathrm{Ab}$ index $)$, calculated as the ratio of sample optical density to cut-off serum mean OD (0.844). The cut-off value was calculated as the product of Calibrator mean OD (1.8775) and Calibrator factor (0.45). The indices below 0.9 were considered negative for IgM specific Antibodies, those above 1.1 were considered positive and those from 0.9-1.1 were considered borderline positive.

\section{Results and Discussion}

A total of 82 patients were tested for the presence of Mycoplasma pneumoniae IgM. Of this total, 2 were positive between 0 and 10 years of age, 7 were positive between 11 and 20 years of age and 1 was positive between 41 and 50 years. Subjects were grouped into following age categories (in years): 0-10, 11-20, 21-30, 31-40, 41-50, 5160, 61-70, 71-80 (Table - 1). IgM antibodies were detected in $0 \%$ to $12.19 \%$ in these groups; the positive rate was higher in children, adolescents and young adults. Results showed highest positive IgM reactivity in the group aged 11-20 years. Overall IgM positive levels were $12.19 \%$ with Calbiotech Mycoplasma pneumoniae IgM ELISA kit. 
Many bacterial and viral infections often share clinical features and symptoms which are difficult to distinguish clinically. Typical clinical features of Mycoplasma pneumoniae include pharyngitis, sore throat, hoarseness and fever. In children under 5 years of age progression to pneumonia is uncommon. Whereas children under 5-15 years of age are likely to develop bronchopneumonia requiring hospitalisation. Apart from respiratory infections it can also cause a wide spectrum of non-pulmonary manifestations like neurological, hepatic, cardiac, hemolytic anaemia and polyarthritis. Of the non-pulmonary manifestations, neurological manifestations are common.

In our study, overall $\operatorname{IgM}$ positive levels were $12.19 \%$. Majority $(8.53 \%)$ of the cases were found in the age group of 11-20 years. Two cases $(2.43 \%)$ were positive under the age group of 10 years. Only one case $(1.21 \%)$ was positive in the adult age group of 41-50 years showing concurrence with other studies. $^{3}$

Hence a sensitive and effective method of detecting these agents is required so that the correct treatment is offered and unnecessary use of antibiotics can be avoided. ${ }^{5}$ Several commercial $M$. pneumoniae antibody detection kits that use PAAA or EIA are available to identify $M$. pneumoniae in human sera. Among the various immunoassays, EIAs are the most widely used in clinical laboratories. ${ }^{6}$

Table.1 Test Results of M.Pneumoniae Igm-Specific Immunoassays by Age

\begin{tabular}{|l|l|l|l|}
\hline Age (Years) & Negative & Equivocal & Positive \\
\hline $0-10$ & $15(18.29 \%)$ & $0(0.0 \%)$ & $2(2.43 \%)$ \\
\hline $11-20$ & $17(20.73 \%)$ & $0(0.0 \%)$ & $7(8.53 \%)$ \\
\hline $21-30$ & $20(24.39 \%)$ & $0(0.0 \%)$ & $0(0.0 \%)$ \\
\hline $31-40$ & $10(12.19 \%)$ & $0(0.0 \%)$ & $0(0.0 \%)$ \\
\hline $41-50$ & $5(6.09 \%)$ & $0(0.0 \%)$ & $1(1.21 \%)$ \\
\hline $51-60$ & $2(2.43 \%)$ & $0(0.0 \%)$ & $0(0.0 \%)$ \\
\hline $61-70$ & $1(1.21 \%)$ & $0(0.0 \%)$ & $0(0.0 \%)$ \\
\hline $71-80$ & $2(2.43 \%)$ & $0(0.0 \%)$ & $0(0.0 \%)$ \\
\hline Total & $72(87.8 \%)$ & $0(0.0 \%)$ & $10(12.19 \%)$ \\
\hline
\end{tabular}

One study from France suggested that positive $\operatorname{IgM}$ and $\operatorname{IgG}$ results varied according to the kit used and the age of the patients ${ }^{7}$.

Our study also demonstrated that age had a major influence on the fraction of positive sera (Table 1). The highest IgM-positive rate occurred in the age group of 11-20 years.

In this age group the manifestatons of M.pneumoniae are more severe like bronchopneumonia of one or two lobes.

This suggests strongly that single-IgM measurement may be helpful in effort towards early diagnosis of $M$. pneumoniae infection among cases of community acquired pneumonia. There by ensuring correct treatment and preventing progression of the disease to severe complication like bronchopneumonia. Therefore, serological test like ELISA should be considered for diagnosing $M$. 
pneumoniae infection in its early phase especially in patients of 11-20 year age group. ${ }^{6}$

In conclusion, the present study has shown that, serological test such as, detection of IgM antibodies by ELISA for precise and reliable diagnosis of Mycoplasma pneumoniae infections during the acute phases of disease is necessary and should be considered as a valid diagnostic approach in early detection of Mycoplasma pneumoniae infections in patients with Community acquired pneumonia. ${ }^{2}$

\section{References}

1. Acharya V,Sahoo R, Shenoy S M, Antony S, Anand R. Study of isolation of Mycoplasma pneumoniae in asthmatics by sputum culture.Lung India 2005;22:50-53.

2. Medjo B, Atanaskovic-Markovic M, Radic S, Nikolic D, Lukac M, Djukic S. Mycoplasma pneumoniae as a causative agent of community-acquired pneumonia in children: clinical features and laboratory diagnosis. Italian J Pedia 2014, 40:104.
3. Kashyap S, Sarkar M. Mycoplasma pneumonia: clinical features and management. Lung India 2010,7(2):7585.

4. Qu J, Cao B. Research progress in atypical pathogens of community acquired pneumonia. Community Acquir Infect. 2014;1:11-4.

5. Kim MH, MD, Kang SY, MD, Lee WI, MD. Comparison of Two Enzyme Immunoassays for Detecting Mycoplasma pneumoniae. Lab medicine. 2012, 43:74-77.

6. Chaudhry R, Sharma S, Javed S, Passi K, Dey AB, Malhotra P. Molecular detection of Mycoplasma pneumoniae by quantitative real-time PCR in patients with community acquired pneumonia. Indian J Med Res, 2013, 138: 244-251.

Petitjean J, Vabret A, Gouarin S, Freymuth F. Evaluation of Four Commercial Immunoglobulin G (IgG)- and IgMSpecific Enzyme Immunoassays for Diagnosis of Mycoplasma pneumoniae Infections. J Clin Microbiol 2002, 40:165-171.

\section{How to cite this article:}

Pramod Sambrani, N., B. Asha Patil and Divya, A. 2016. Study of Prevalence of Community Acquired Mycoplasma pneumoniae Infections at KIMS Hospital, Hubballi, India. Int.J.Curr.Microbiol.App.Sci.5(2): 707-710. doi: http://dx.doi.org/10.20546/ijcmas.2016.502.078 